\title{
SEX DIFFERENCES IN ANDROGEN ACTIVATION OF COMPLEX COURTSHIP BEHAVIOR
}

\section{ABSTRACT}

Sexual dimorphism is common but evolutionarily labile in vertebrates. While it is well established that gonadal hormones exert considerable influence on the development and expression of sexual traits, studies of animals with exceptional sexually dimorphic neural or neuromuscular phenotypes are limited. We studied the extent to which androgen treatment of female golden-collared manakins (Manacus vitellinus) activates specific elements of the elaborate, acrobatic courtship behavior characteristic of males. After one week, non-breeding females and juvenile males given implants containing testosterone (T) were observed for 3 weeks in an outdoor aviary situated in the middle of Panamanian rainforest. T-treated males performed the full suite of documented masculine courtship behaviors, whereas T-treated females performed only a few of these behaviors and then at much lower rates than males. T- treatment did increase aggressive behavior to a similar degree in both males and females. These results suggest that neuromuscular systems encoding elements of male courtship as well as aggressive behavior experience unique patterns of development from complete to limited to non-existent sexual differentiation. The basis of these patterns represents a unique opportunity for study.

Keywords: courtship; mating behavior; testosterone; hormonal activation; sexual differentiation; manakin. 


\section{INTRODUCTION}

25

26 Males and females share most of their genome and this is expected to constrain the

27 evolution of sexually dimorphic traits (Ellegren and Parsch 2007; Cox and Calbeek

28 2009). In spite of this, sexual dimorphism is common and evolutionarily labile (Wiens

29 1999; Price et al. 2007; Odom et al. 2014). In vertebrates, two mechanisms appear to

30 mediate the development of sex-specific phenotypic traits with one linking the expression

31 of shared loci to gonadal hormones (Phoenix et al. 1959; Adkins-Regan 2012) and the

32 other stemming from expression of sex-specific loci arising from their position on sex

33 chromosomes (Arnold 2009; McCarthy 2016). Gonadal-hormone dependent behavioral

34 sexual differentiation is well established. In mammals, testosterone (T) circulates at

35 greater levels in males during early development to organize the masculine neural

36 circuitry, which is then activated in adulthood to enable male-specific behaviors to occur

37 in the presence of T. By contrast, in birds ovarian estrogens demasculizine female circuits

38 rendering them fully or partially insensitive to $\mathrm{T}$ such that experimentally increasing $\mathrm{T}$

39 levels in adult females activates few or little if any masculine behavior (Lank et al. 1999;

40 Lahaye et al. 2012; Madison et al. 2015).

41

42 Organizational and activational effects of $\mathrm{T}$ are species, behavior, and tissue specific

43 (Adkins-Regan 2012; McCarthy 2016). In the case of behavior, traits may be part of

44 different 'modules', allowing them to evolve independently in the face of sexual selection

45 pressures and thus to differ in the extent to which they acquire steroid-sensitivity or fall

46 under sex-chromosome dependent control (West-Eberhard 2003). These levels of control 
47 likely depend on whether these traits are expressed exclusively in a reproductive context,

48 like copulatory behaviors, or can be expressed in non-reproductive contexts, like

49 aggressive or singing behaviors (Kendrick and Schlinger 1996; Schlinger 1998; Adkins-

50 Regan 2012).

51

52 Courtship behaviors can be striking composites of reproductive and non-reproductive

53 behavioral elements, or modules (Huxley 1914; Tinbergen 1951), and thus, courtship

54 behaviors might be organized developmentally in diverse ways involving different modes

55 of hormone-dependence or sex-chromosomal control (Arnold 2009; Schlinger 1998;

56 Adkins-Regan 2012). Moreover, whereas courtship behavior is usually expressed only

57 by males and serves as a basis for mate-choice by females, in some species, both males

58 and females participate together in such displays, especially as a mechanism for

59 establishment of pair-bonds (Langmore 1998). Thus, the neural and muscular machinery

60 underlying the motivation and capacity to perform courtship displays are subject to

61 unique selection pressures that make them available for capture by hormone-independent

62 or hormone-dependent organizational/activational mechanisms.

63

64 To understand the degree to which aggressive and courtship behaviors are expressed in

65 adults in a sex-specific pattern, we have examined the extent to which T can activate in

66 females several male-typical behaviors of golden-collared manakins (Manacus vitellinus;

67 hereafter GCmanakin). Many species of manakins (family Pipridae) are characterized by

68 polygynous mating systems in which males aggregate at leks where they defend

69 individual courts and perform complex courtship displays (Prum 1990). Male 
70 GCmanakins perform an exquisitely acrobatic courtship routine with multiple display

71 elements (Chapman 1935). Conspicuously, males produce wingsnaps created by

72 forcefully beating their wings together above their heads to produce loud clapping

73 sounds. Wingsnaps are produced singly, in mid-flight, or as 'rollsnaps' which consist of a

74 series of $17-25$ wing-snaps produced rapidly $(50-60 \mathrm{~Hz})$ in sequence. Acrobatic features

75 of the courtship dance, or jump-snap display, includes males swiftly leaping between

76 saplings when the single wing-snap is produced midflight. Males also perform a

77 jumpsnap with a half-twist landing on the ground followed by a grunt-jump back onto a

78 sapling. The last move involves flying vertically to a perch with the 'grunt' sound

79 produced by flapping of the wings (Bostwick and Prum, 2003). Thus, manakin courtship

80 displays utilize a variety of neuromuscular systems for use in a mating context (Fuxjager

81 and Schlinger 2015). To date, little is known about the extent that these elements may be

82 developmentally organized differently between the sexes or whether differences in adult

83 androgen levels maintain sexual dimorphism in courtship behavior.

84

85 Previous studies of wild male GCmanakins show that circulating $\mathrm{T}$ is elevated during the

86 breeding season (Day et al. 2006; Schlinger et al. 2013) and that exogenous T, acting

87 through androgen-receptors (AR), activates courtship behaviors in non-breeding and

88 juvenile males with low circulating T levels (Fuxjager et al. 2013). Interestingly, female

89 GCmanakins have little circulating $\mathrm{T}$ but they express AR at levels comparable to those

90 of males in skeletal muscle and spinal cord (Feng et al. 2010; Fuxjager et al. 2012). Thus,

91 it is plausible that androgens could activate these neuromuscular systems to produce

92 'male-like' behavior. Females do join males in their jump-snap display before allowing 
93 some males to copulate, but they are not known to perform wingsnaps, rollsnaps or to

94 jump (they only fly) between saplings with or without males. In small cages, T-treated

95 females do occasionally produce wing- and roll-snaps (Day et al. 2007). Thus, females

96 and males may overlap in the development of at least some neuromuscular systems

97 controlling courtship display.

98

99 In this study, we observed females and juvenile males treated with or without $\mathrm{T}$, in a large

100 tropical forest aviary that permits the full range of behaviors under highly natural

101 conditions. We asked whether T activates the entire repertoire of courtship elements in

102 juvenile males and whether females perform some or all of the male's behavioral

103 repertoire.

104

105

106 METHODS

107

108 The work was conducted at the Smithsonian Tropical Research Institute (STRI) in

109 Gamboa, Panama. Experiments were conducted during the non-breeding season (July to

110 mid-Dec) in both 2014 and 2015. During this season, lek activity is considerably reduced

111 compared to the breeding season (Schlinger et al. 2013) and circulating T levels are basal

112 or near so in all birds (Day et al. 2006). Moreover, T-treatment of juvenile males at this

113 time effectively activates several elements of adult courtship, such as wing-snaps and

114 rollsnaps (Day et al. 2006, 2007). GCmanakins were captured using passive mist netting,

115 individually marked with plastic colored leg-bands, weighed, and transferred to STRI 
116 facilities in Gamboa, where they were housed individually in cages $\sim 36 \times 29 \times 32 \mathrm{~cm}$.

117 Birds were in visual and auditory contact in cages set on a table in a room with open

118 screened windows (allowing ventilation and natural lighting). Supplemental lighting was

119 added and timed to coincide with the ambient sunrise and sunset. Fresh papaya with

120 vitamin supplements and water were provided twice daily (ad libitum).

121

\section{Ethical Note}

123 The work was conducted under permits from local Panamanian authorities (Autoridad

124 Nacional del Ambiente and MiAmbiente permits SE/A-4-14 and SE/A-55-15) and in

125 accordance with animal care policies at the Smithsonian Tropical Research Institute

126 (Animal Care and Use Committee Protocol number 2013-0315-2016) and at UCLA

127 (Office of Animal Research Oversight, ARC Protocol number 2009-123-21), which

128 adhere to the standards set by ASAB/ABS (2012). All birds were released at the point of

129 capture following the end of experiments.

130

131 Sex determination

132 Females and juvenile males are superficially indistinguishable from one another bearing

133 an overall olive-green coloration. Thus, we determined each bird's sex using a molecular

134 approach that involves amplifying homologous sections of the CHD gene, which is

135 present on both $\mathrm{Z}$ and $\mathrm{W}$ chromosomes, but which differs in length. Products appear as

136 one or two bands respectively for males and females (Griffiths et al. 1998). After capture,

$137 \sim 5-20 \mu \mathrm{L}$ of blood was collected by puncture of the brachial vein and stored in $100 \mu \mathrm{L}$

138 Queen's lysis buffer at $4^{\circ} \mathrm{C}$ until analysis. DNA was extracted using the Sigma Aldrich 
139 DNeasy Blood and Tissue kit and the concentration was adjusted to $20 \mathrm{ng} / \mu \mathrm{L}$. The CHD140 gene was PCR-amplified (2.5 $\mu \mathrm{L}$ DNA solution, $3.0 \mu \mathrm{L}$ of $20 \mathrm{mM} \mathrm{MgSO}_{4}, 1.0 \mu \mathrm{L}$ of $10 \mathrm{X}$

141 buffer solution, $2.9 \mu \mathrm{L}$ water, $0.2 \mu \mathrm{L}$ dNTPs solution, $0.2 \mu \mathrm{L}$ of each $\mathrm{P} 2$ and P8 primers, $1420.05 \mu \mathrm{L}$ Taq Master Mix (Qiagen)) using the following protocol: 2 min denaturing step at $14394^{\circ} \mathrm{C}$ followed by 35 cycles of $20 \mathrm{~s}$ at $72^{\circ} \mathrm{C}, 20 \mathrm{~s}$ at the annealing temperature, $62^{\circ} \mathrm{C}$, and $14430 \mathrm{~s}$ at $72^{\circ} \mathrm{C}$. PCR products were separated by electrophoresis for $45-60 \mathrm{~min}$ at $60 \mathrm{~V}$ in a $1453 \%$ gel stained with GelRed (Biotium Inc.). Samples were run in duplicate together with 146 DNA from 1-2 known sex males or 1known male and 1 known female.

147

148 T-implant administration

149 Once 3-4 same sex birds were available, they were implanted at the base of the neck with 150 silastic tubes (Dow Corning Inc., 10mm long, $0.76 \mathrm{~mm}$ inner diameter, and $1.65 \mathrm{~mm}$ outer 151 diameter) filled with crystalline T (Steraloids Inc.) or empty as control. Details of the 152 implantation procedure were described previously (Fuxjager et al. 2012) and show that 153 levels of T were elevated in females on average to $2.24 \mathrm{ng} / \mathrm{ml}$ as compared levels in 154 unimplanted females of $0.51 \mathrm{ng} / \mathrm{ml}$. In separate studies using different implants, male155 like behaviors were detected in T implanted birds 7 days post-treatment and peaked at 156 day 13 (Day et al. 2007), so here, implanted birds were relocated to the outdoor aviary 7 157 days following implantation where they were observed for 21 days ( 17 days for one 158 group, see below). 
162 Aviary setup

163 The outdoor aviary (Appendix fig. A1) is a $10 \mathrm{~m} \times 5 \mathrm{~m} \times 4.5 \mathrm{~m}$ tall enclosure located $~ 2$

$164 \mathrm{~km}$ inside Soberania National Park near the town of Gamboa. It has a $0.5 \mathrm{~m}$ cement base

165 with chain link fence walls and ceiling covered on the inside with soft plastic mesh

$166(1 \mathrm{~cm})$. A sheet of corrugated steel covers $\sim$ one-third of the enclosure, including over a

$1671 \mathrm{~m}^{2}$ double-door entrance area. The base was filled with soil in which we planted 45

168 small saplings $(<2 \mathrm{~m})$ with straight, unbranching stems preferred by males for

169 performing courtship displays, as well as 7 trees (2-4 $\mathrm{m}$ tall) that the birds use to rest.

170 Fresh papaya and banana slices with liquid vitamin supplement were provided at four

171 ant-proof and rain-protected feeders. To ensure feeding on the first day of introduction,

172 additional papaya was presented on skewers throughout the aviary as well as some

173 berries (Miconia or Psychotria spp.), typical food of manakins in the wild. Fresh food

174 was provided twice a day, in the morning and at midday. A water bath was provided,

175 cleaned and refilled daily.

176

177 Behavioral observations

178 We observed a total of 3 groups of T-treated individuals, 2 groups of females (4 birds,

179 from Oct 9-25 2014; and 3 birds, from Nov 19 to Dec 9, 2015) and one group of juvenile

180 males (4 birds, from Nov 25 to Dec 14, 2014). Observations of the first T-treated group of

181 females was terminated after 17 days (all others 20-21 days) because the aggressiveness

182 of one female prevented the other females from accessing the feeders. During the second

183 experiment with T-treated females, one female was also more aggressive than the others, 
184 which restricted their use of feeders, so we added 8-10 feeding locations (skewer feeders)

185 throughout the aviary, which allowed the other females access to food in spite of

186 increased agonistic interactions. We also observed two groups with blank implants, one

187 group of females (4 birds, Sept 1-21, 2015) and one group of males (3 birds, Oct 8-28, 188 2015).

189

190 Birds were introduced to the aviary between 8:30AM and 12:00PM and observed for 1-2

191 hrs to ensure they fed. Afterwards, $4 \mathrm{hrs}$ of behavioral observations were performed daily

192 after morning food provision at 6:30AM. The observer sat in the double door entrance.

193 A tripod-mounted Sony Handycam CX220, set near the entrance, recorded each

194 observation period, capturing activity in $\sim 70 \%$ of the aviary. We documented the

195 occurrence of previously described behaviors (Chapman 1935; Day et al. 2006, 2007;

196 Schlinger et al. 2013): 1) rollsnaps, which consist of a series of quick wing-snaps; 2)

197 jumpsnap displays, which consist of hopping from one sapling to another while wing-

198 snapping midflight; we included here jumps with no wing-snapping, which may be rarely

199 performed by males just prior to launching into a jump-snap display; 3) half-flip jumps to

200 the ground and grunt-jump displays; and 4) arena cleaning, which involves removing

201 leaves or other dead plant materials from the court or biting and pulling at shoots or

202 leaves of live plants. To ensure that plant material of different types was available for

203 removal, and because leaves can become covered in mud during the rainy season, we

204 augmented dead leaves throughout the aviary, twice during each experiment ( every 7

205 days from the experiment's onset). 
207 Typically in lekking animals, males compete for access to females. In GCManakins this

208 involves gaining dominance for possession of a court so courtship displays can be

209 performed without interruption during female visits (see Foster 1981). We examined the

210 frequency of displacements and chases (agonistic behaviors), both on and away from the

211 court (including at feeders) as all likely contributed towards establishment of dominance.

212 A displacement involved one bird being replaced by another on a perch (or less

213 frequently leaving the perch in anticipation of another landing nearby) without leaving

214 the immediate area. Chases were defined as one bird pursuing another in flight,

215 sometimes protracted and culminating in a fight. Male GCManakins also produce snips

216 infrequently, which are quieter wing-snaps that can be performed while perched or while

217 individuals hop from branch to branch while chasing each other at the lek (Chapman

218 1935). Thus we recorded their occurrence for both sexes and treatments.

220 Statistical analysis

221 Some behaviors were performed only by males and statistics were not used for

222 comparison with females. Females performed some recorded behaviors (rollsnaps, jump

223 displays, snips) infrequently and due to many zero values, we were unable to transform

224 the data to attain normality. We therefore used mixed-effects logistic regression analyses

225 to determine whether T-treatment increased the probability of females performing male-

226 like behaviors. Secondly, to explore the effects of T-treatment on the rate of behavioral

227 performance (the number of occurrences observed per day), we modeled the subset of

228 observations that were greater than zero using a generalized linear mixed-effects model.

229 Since data were counts of observations, we used a Poisson or negative binomial error 
230 distribution (if the data were overdispersed). For both types of analyses, we modeled each

231 behavior separately and included sex, treatment, day in the aviary, and day x treatment

232 interaction as factors with bird id as a random variable. For each behavior, we initially

233 included all factors in the model and assessed the contribution of each of the variable by

234 comparing models with and without each variable using a chi-square test. If sex or

235 treatment were significant predictors, we then performed simplified models that included

236 only 2 of the 4 groups (control-females, T-females, control-males, T-males) at a time and

237 this allowed us to determine which pairs showed significant differences in daily

238 probability of behaviors. Blank-treated females did not perform some of the behaviors of

239 interest (rollsnaps, jump-displays, snips, or chases), therefore, models examining

240 differences in daily rates only included 3 groups (T-females, control-males, and T-males)

241 in these cases. All statistical analyses were conducted in R, version 3.3.0.

242

243

\section{RESULTS}

245

246 During a pilot study (March 1 - May 15, 2014), we assessed the utility of the aviary for

247 studies of GCmanakin behavior by releasing three T-implanted juvenile males into the

248 aviary. Within 24 hrs of introduction, two birds performed jumpsnap displays. Daily

249 observations (15-30 $\mathrm{min})$ indicated that all had established arenas and performed

250 courtship displays most days we visited. The aviary is located within forest near $(\sim 10-$

251 20m) an established lek of wild GCmanakins. Occasionally, adult males and green-

252 plumaged birds were attracted to the aviary stimulating captive males to call in response. 
253 These observations gave us confidence to proceed with further investigations of courtship

254 display and social behavior in this semi-natural setting.

255

256 Courtship display behavior

257 Juvenile males with blank implants initiated courtship behaviors starting on day 3 of 258 observations, whereas males treated with $\mathrm{T}$ initiated display on day 1 . Juvenile males in

259 both treatments performed the full extent of the courtship display seemingly

260 indistinguishable from that of adult males (Supplementary material video). The typical

261 courtship routine starts with 1-2 jumps between saplings with no wing-snap midflight,

262 followed by jump-snaps, and occasionally a half-flip jump and a grunt-jump display

263 element. For juvenile males, jumps not accompanied by wing-snaps were infrequent (<

$2644 \%$ of total jumps based on 1243 jump/jump-snap displays by 3 males observed over 5

265 days), so we used the total number of jumps and jump-snaps in our analysis of display

266 behavior.

267

268 When treated with T, females also showed some male-like behaviors starting on day 2 of

269 observations whereas no courtship behaviors were observed in blank-treated females.

270 Five of the 7 females performed rollsnaps, one performed jumps between saplings close

271 to the ground $(<1 \mathrm{~m})$ and another 4 performed jumps between branches $>1 \mathrm{~m}$ in trees.

272 None of the females performed jump-snaps, or jumps/jump-snaps landing on the ground,

273 or half-flip jumps or grunt-jump displays. Also notably, when females performed jumps,

274 they flapped their wings midflight, thus this behavior differs from that of adult males

275 jumping between saplings, as adult males do not flap their wings (Fusani et al. 2007). 
276 Nevertheless, our observations (including examination of video recordings at 30 frames 277 per second) indicate that juvenile males also flap their wings during some jumps that are 278 not accompanied by a wing-snap. Female rollsnaps were quieter than those of males and 279 shorter; we examined the number of snaps in a rollsnap by inspecting sonograms of audio 280 collected during observations in the aviary (using the software Audacity 2.0.0), and for 281 females, rollsnaps contained $3.92 \pm 2.17$ (S.D.) snaps (range of 2 to 10 snaps, $n=26$ 282 rollsnaps) compared to $7.61 \pm 3.12$ (S.D.) snaps $(3-18$ snaps, $\mathrm{n}=71$ rollsnaps $)$ for males 283 (Wilcoxon rank sum test $\mathrm{W}=1611, \mathrm{P}<0.001$ ).

284

285 The probability of performing courtship behaviors in both sexes was significantly 286 impacted by T-treatment (fig. $1 \mathrm{~A}$, rollsnaps $\chi=9.95, \mathrm{P}=0.002$; jumps/jump-snaps: $\chi=$ 287 7.91, $\mathrm{P}=0.004)$. Males were more likely to perform rollsnaps and jump-snap displays 288 compared to females (rollsnaps: $\chi=17.26, \mathrm{P}<0.001$; jumps/jump-snaps: $\chi=29.86, \mathrm{P}<$ 289 0.001). Comparisons of simplified models including only 2 groups at a time (of the 4 290 groups: blank-females, T-females, blank-males, and T-males), indicate that all pair-wise 291 comparisons are significant for jump-displays and rollsnaps (all $\mathrm{P}<0.001$ ). In addition, 292 our results indicate that the probability of individuals performing rollsnaps and 293 jump/jump-snap displays increased significantly with time in the aviary (rollsnaps: $\chi=$ 294 15.81, $\mathrm{P}<0.001, \mathrm{P}<0.001 ;$ jumps/jump-snaps $\chi=26.58, \mathrm{P}<0.001)$. 295 For the subset of days when individuals were observed to perform the behavior of 296 interest, the number of rollsnaps and jump-displays in a day by an individual increased 
297 significantly with $\mathrm{T}$ treatment (fig. $1 \mathrm{~B}$, rollsnap rate: $\chi=3.9, \mathrm{P}=0.048$; jump-snap rate:

$298 \chi=5.04, \mathrm{P}=0.025)$, however only the jump-display rate was significantly different

299 between the sexes $(\chi=20.37, \mathrm{P}<0.001)$. Follow-up comparisons of simplified models

300 including only 2 groups at a time (of the 3 groups: T-females, control-males and T-males)

301 indicate that for jump-snap displays all groups are significantly different from each other

302 (all P < 0.001), whereas for rollsnaps, control males showed no significant difference in

303 daily rate of roll-snaps from T-treated females $(\chi=2.35, \mathrm{P}=0.50)$. T-treated males

304 showed significant differences from T-treated females $(\chi=21.19, \mathrm{P}<0.001)$ and control

305 males $(\chi=25.26, \mathrm{P}<0.001)$.

306 The probability of individuals performing rollsnaps and jump/jump-snap displays

307 increased significantly with time in the aviary (rollsnaps: $\chi=15.81, \mathrm{P}<0.001, \mathrm{P}<0.001$;

308 jumps/jump-snaps $\chi=26.58, \mathrm{P}<0.001)$. The number of jump-displays performed in a

309 day by an individual also showed a significant increase with time in the aviary and

310 similarly, the number of rollsnaps also increased over time in the aviary (fig. $3 \mathrm{~A}$ and B,

311 jump/jump-snaps: $\chi=34.87, \mathrm{P}<0.001$, rollsnaps: $\chi=15.44, \mathrm{P}<0.001$ ), however for

312 rollsnaps, there was an interaction with treatment, such that blank-treated individuals

313 showed a decrease in daily rollsnap rate over time in the aviary $(\chi=4.53, \mathrm{P}=0.033)$. 


\section{Aggressive behaviors and vocalizations}

317 Males in both treatment groups established arenas where they performed most of the

318 recorded display behavior (97\% of 1344 jump-snap displays over 4 days). For females,

319 and particularly for the one that performed jumps between saplings, no court

320 establishment was observed and jumps were performed at different locations throughout

321 the aviary. Nevertheless, the probability of displacements was not different between the

322 sexes or between treatments (fig. $2 \mathrm{~A}$, sex: $\chi=1.33, \mathrm{P}=0.25$, treatment: $\chi=0.40, \mathrm{P}=$

323 0.53). However, the probability of chases increased significantly with T-treatment $(\chi=$

$32416.17, \mathrm{P}<0.001)$ and was higher for males $(\chi=9.48, \mathrm{P}=0.0021)$. Follow-up models

325 including only 2 groups at a time indicate that all groups were significantly different from

326 each other $(\mathrm{P}<0.001)$.

327 The number of displacements by individuals did not differ with treatment or

328 between the sexes (fig $2 \mathrm{~B}$, treatment: $\chi=0.01, \mathrm{P}=0.94$; sex: $\chi=0.10, \mathrm{P}=0.76$ ). The

329 number of chases observed in a day was significantly higher for T-treated females than

330 for males $(\chi=7.49, \mathrm{P}=0.006$ ). Follow-up models including only 2 groups at a time (of

331 the 3 groups: T-females, blank-males, and T-males) showed that T-females differed

332 significantly from $\mathrm{T}$-males $(\chi=4.13, \mathrm{P}=0.042)$ but not from blank-males $(\chi=5.87, \mathrm{P}=$

$3330.053)$.

334 Over the course of observation in the aviary, the probability of displacements did

335 not change $(\chi=2.97, \mathrm{P}=0.085)$, however the probability of chases increased 
336 significantly $(\chi=17.07, \mathrm{P}<0.001)$. The number of displacements also did not change

337 over the observation period but the number of chases showed an increase for T-females

338 relative to both groups of males (fig. $3 \mathrm{C}$ and $\mathrm{D}, \chi=6.49, \mathrm{P}=0.011$ ).

339 The probability of snips increased with T-treatment, however it was not different

340 between the sexes (fig $2 \mathrm{~A}$, treatment: $\chi^{2}=4.83, \mathrm{P}=0.028$, sex: $\chi^{2}=0.12, \mathrm{P}=0.73$ ). The

341 number of snips was also not different with treatment or sex (fig. 2B) and the daily rate of

342 snips was not significantly predicted by time in the aviary(fig. 3E).

343

344

345 DISCUSSION

346 Long-term behavioral studies of wild female golden-collared manakins are challenging

347 because they disperse after release following capture and their dull-green plumage makes

348 them cryptic in the forest they inhabit. Thus, a major objective of this study was to

349 provide suitable captive conditions that would promote our ability to study female

350 behavior and the possible expression of the male behavioral phenotype after treatment

351 with testosterone.

352 In the absence of $\mathrm{T}$, female GCmanakins performed no male-typical courtship displays.

353 Following treatment with T, females still did not claim a court, clear an arena (the subject

354 of a follow-up study), perform half-flip jumps or grunt-jump displays. Females treated

355 with T did, however, perform several male-typical courtship elements, usually at lower

356 rates than juvenile males. Females performed single wing-snaps while perched (a 
357 behavior rarely observed in males), as well as rollsnaps and jumps without wingsnaps. In

358 particular, in the absence of a male, jumps have not previously been reported for females

359 with or without T-treatment. Thus, whereas females displayed some motivation to engage

360 in male courtship, either they were insufficiently motivated or physically incapable of

361 performing the full suite of male behaviors, even in the presence of elevated plasma T.

362 Juvenile males, with and without T-treatment, performed the complete courtship display,

363 which includes complex moves such as jump-snaps, grunt jumps, and half-flip jumps.

364 Thus, our observations indicate that female treatment with $\mathrm{T}$ results in partial expression

365 of male courtship display elements. This indicates that some neuromuscular systems

366 supporting courtship behavior are present in females and are partially coupled to T.

367 Nevertheless, some male-like neuromuscular tissues are, in females, either insensitive to

368 T or are absent altogether. These results indicate that there is partial developmental sexual

369 differentiation of the neuromuscular systems involved in display behavior. Females

370 treated with $\mathrm{T}$ were as aggressive as males, suggesting that the development of

371 neuromuscular components supporting aggression may differ from those supporting

372 courtship and copulatory behaviors.

373

374 Courtship

375 An initial point of interest is that juvenile males perform male courtship during the non-

376 breeding season when T levels are basal. While we have seen evidence for this before

377 (Day et al. 2006), these observations document quite clearly that both central

378 motivational and peripheral neuromuscular systems are relatively intact and functional in 
379 these males. Males probably develop their adult plumage after their $2^{\text {nd }}$ year, so these 380 males could have been a few months to over a year in age. Thus, it is possible that some 381 males had experienced elevated levels of $\mathrm{T}$ at earlier months and/or had some time to 382 practice courtship routines. Nevertheless, T-treatment significantly amplified the overall 383 production of these behaviors. In skeletal muscles involved in courtship, androgens 384 significantly upregulate genes encoding proteins involved in energy utilization (Fuxjager 385 et al. 2016), thus one main function of androgenic activation of male courtship may lie in 386 the regulation of optimal muscle metabolism required for sustained behavioral 387 performance.

388 Conspicuously, female GCmanakins naturally perform little that resembles male-typical 389 courtship, even here in the presence of $\mathrm{T}$ that effectively activated courtship behavior in 390 males and that activated aggressive behavior in females. In female GCmanakins, the 391 activation of male courtship behaviors seem to fall into two categories, those never 392 performed by females and those performed by females, but significantly less frequently 393 than by males. With or without $\mathrm{T}$, females seem to have no motivation to establish a court 394 and keep it cleared of debris. Presumably, this failure to claim a court represents a fully 395 sexually-dimorphic motivational system, one present in males, but not in females. 396 Females also never perform the half-flip to the ground and grunt-jump back to a perch. 397 When a female has joined a male in his courtship dance, this latter behavior is often 398 performed by the male just prior to copulation, so its performance by males may be a 399 component of copulatory behavior per se, and thus subject to gonadal hormone400 dependent organizational mechanisms as are sex-specific copulatory behavior in other 
401 species (Balthazart et al. 1996; Adkins-Regan 2012). In GCManakins it has become one

402 element in the male's courtship display, performed with or without a female partner.

403 We cannot dismiss the idea that T-treated females might have displayed male copulatory

404 behaviors had they been housed together with non T-treated, and thus possibly receptive,

405 females. Some females did, however, clearly dominate others, and they might have

406 attempted to court and copulate with these subordinate females. Over many hours of

407 observations, our data suggest that copulatory behavior, as in adults of other species, is

408 fully sexually dimorphic (Adkins-Regan 2012) unlike perched snaps and rollsnaps that

409 can be activated by $\mathrm{T}$ in females.

410 Although performed less frequently, T-treated females did perform both rollsnaps (5 of 7

411 females) and one performed jumps between saplings, though in no single location as is

412 done by males. It appears that females are unable to perform the midflight single

413 wingsnap, unless perhaps considerable practice is involved requiring longer-term T-

414 treatment. Some females did produce rollsnaps, a complex behavior. Presumably,

415 females possess the full androgen-dependent neuromuscular system (and forelimb

416 anatomy, Bodony et al. 2016) to achieve this high degree of forelimb neuromuscular

417 speed and accuracy required to produce these behaviors, even when they seldom, if ever,

418 naturally perform this behavior. 


\section{Aggressive behaviors}

423 Females were aggressive, displacing other females throughout the aviary as often as did

424 males. With T-treatment, females showed an increase in the level of aggressive behavior,

425 specifically chases, such that the number per day was higher than that displayed by

426 males. T-treated females also produced snips, which in males are observed in male-male

427 interactions near the court (Chapman 1935). However, no females showed behavior that

428 resembled establishment and defense of a specific court suggesting female aggression

429 was not driven by the motivation to establish a court. In females, snips might function

430 under circumstances unrelated to mate competition, such as part of a distraction display

431 when defending a nest (see Bodony et al. 2016). Observations of female behavior in the

432 wild are limited, however they do not appear normally aggressive, so our results were a

433 surprise. The data suggest that female manakin aggressiveness requires additional

434 investigation. The data do point to a specific T-dependent activation of aggression in

435 females, suggesting that the underlying neural circuits involved in aggressive motivation

436 and/or motor output are sensitive to this hormone in female GCmanakins, as they are in

437 males. Thus, developmental mechanisms organizing neural circuits controlling

438 aggression differ from those underlying most courtship display and copulatory-related

439 behavioral elements.

440

441 General conclusions and discussion 
442 The many notable elements of male GCmanakin social behavior, their creation,

443 maintenance and territoriality around a courtship arena, the performance of their

444 elaborate, acrobatic courtship display and their production of acoustic signals using both

445 syringeal-based (vocal) and forelimb-based (wingsnap) mechanisms, appear to express

446 varying degrees of sexual dimorphism in response to T-activation (Table 1). Previous

447 studies show that both males and females possess a radius morphology unique among

448 birds, and that appears to be the source of the wingsnap sound (Bodony et al. 2016). In

449 addition, male and females both possess forelimb muscles that exhibit elevated AR

450 expression (Feng et al. 2010) upon which T acts to facilitate the production of wingsnaps

451 (Fuxjager et al. 2013). Thus, these birds have evolved the peripheral neuromuscular

452 capacity for hormone-dependent control of wingsnap production. The fact that the

453 probability of wingsnap and rollsnap production differs across the sexes, likely stems

454 from differences in central motivation centers and the degree to which androgens activate

455 those circuits. It is possible that wingsnaps evolved originally for non-reproductive

456 purposes (such as for contact maintenance between individuals or for predator

457 deterrence) and were later incorporated into the male's courtship display, an evolutionary

458 perspective on courtship applied previously (Tinbergen 1951).

459 In contrast to these behaviors, females appear incapable of expressing some male-typical

460 behaviors either because central motivational circuits are lacking or are $\mathrm{T}$ insensitive.

461 These latter behaviors show substantial overlap with those behavioral elements that

462 appear to provide unique information to females about male quality (Fusani et al. 2007; 
463 Barske et al. 2011) and thus developed exclusively in males by independent mate choice 464 pressures.

465 Neuroanatomical studies reveal characteristics that may underlie some of these

466 behavioral phenotypes. Males exhibit greater aromatase activity in the hypothalamus

467 pre-optic area than do females (Saldanha et al. 2000), a pattern consistent with other

468 species where estrogens promote masculine copulatory behaviors (Balthazart et al. 1996).

469 Whereas we have found a prominent role for androgens, and not estrogens, in the

470 activation of GCmanakin male courtship (Schlinger et al. 2013), we cannot dismiss the

471 possibility that estrogens promote some male behavioral elements. The arcopallium is

472 larger in males than females and this is a likely site for pre-motor control of some display

473 elements (Day et al. 2011). Additional work is required to establish just how different

474 brain regions participate in controlling the discrete elements of courtship. Moreover,

475 hormonal manipulations of GCmanakin embryos are needed to elucidate how sex

476 differences, where they exist, are produced and lead to the complex sexually dimorphic

477 and hormone-dependent behavioral phenotype we observe.

478

479

480 REFERENCES

481 Adkins-Regan, E. 2012. Hormonal organization and activation: Evolutionary

482 implications and questions. General and Comparative Endocrinology 176: 279-

483285. 
484 Arnold, A. P. 2009. The organizational-activational hypothesis as the foundation for a 485 486 487 488 ASAB/ABS. 2012. Guidelines for the treatment of animals in behavioural research and teaching. Animal Behaviour, 83, 301-309.

489

490

491

492

493

494

495

496

497

498

499

500

501 502 unified theory of sexual differentiation of all mammalian tissues, Hormones and Behavior 55, 570-578.

Balthazart, J., O. Tlemçani, and G. F. Ball, 1996. Do sex differences in the brain explain sex differences in the hormonal induction of reproductive behavior? What 25 years of research on the Japanese quail tells us. Hormones and Behavior 30, 627661.

Barske, J., B. A. Schlinger, M. Wikelski, and L. Fusani. 2011. Female choice for male motor skills. Proceedings of the Royal Society of London B: Biological Sciences

Bodony, D. J., L. Day, A. R. Friscia, L. Fusani, A. Karon, G. W. Swenson, et al. 2016. Determination of the wingsnap sonation mechanism of the Golden-collared manakin (Manacus vitellinus). Journal of Experimental Biology 219:1524-1534.

Bostwick, K. S. and R.O. Prum. 2003. High-speed video analysis of wing-snapping in two manakin clades (Pipridae: Aves). Journal of Experimental Biology 206:36933706.

Chapman, F.M. 1935. The Courtship of Gould's Manakin (Manacus vitellinus vitellinus) on Barro Colorado Island, Canal Zone The Auk 53:111-112. 
503 Cox, R.M. and R. Calsbeek. 2009. Sexually antagonistic selection, sexual dimorphism, 504 and the resolution of intralocus sexual conflict. American Naturalist 173: 176-187.

505 Day, L.B., L. Fusani, E. Hernandez, T.J. Billo, K.S. Sheldon, P.M. Wise, et al. 2007.

506 Testosterone and its effects on courtship in golden-collared manakins (Manacus

507 vitellinus): Seasonal, sex, and age differences. Hormones and Behavior 51:69-76.

508 Day, L. B., L. Fusani, C. Kim, and B.A. Schlinger. 2011. Sexually Dimorphic Neural 509 Phenotypes in Golden-Collared Manakins (Manacus vitellinus). Brain Behav. $510 \quad$ Evol. 77:206-218.

511 Day, L. B., J. T. McBroom, and B. A. Schlinger. 2006. Testosterone increases display 512 behaviors but does not stimulate growth of adult plumage in male golden-collared 513 manakins (Manacus vitellinus). Hormones and Behavior 49:223-232.

514 Ellegren, H. and J. Parsch. 2007. The evolution of sex-biased genes and sex-biased gene 515 expression. Nat. Rev. Genet. 8:689-698.

516 Feng, N. Y., A. Katz, L. B. Day, J. Barske, and B. A. Schlinger. 2010. Limb Muscles Are 517 Androgen Targets in an Acrobatic Tropical Bird. Endocrinology 151:1042-1049.

518 Foster, M. S. 1981. Cooperative behavior and social organization of the Swallow-tailed 519 Manakin (Chiroxiphia caudata). Behavioral Ecology and Sociobiology 9:167$520 \quad 177$. 
521 Fusani, L., M. Giordano, L. B. Day, and B. A. Schlinger. 2007. High-Speed Video 522 Analysis Reveals Individual Variability in the Courtship Displays of Male 523 Golden-Collared Manakins. Ethology 113:964-972.

524 Fuxjager, M. J., J. Eaton, W. R. Lindsay, L. H. Salwiczek, M. A. Rensel, J. Barske, et al. 525 2015. Evolutionary patterns of adaptive acrobatics and physical performance 526 predict expression profiles of androgen receptor - but not oestrogen receptor - in 527 the forelimb musculature. Functional Ecology 29:1197-1208.

528 Fuxjager, M. J., K. M. Longpre, J. G. Chew, L. Fusani, and B. A. Schlinger. 2013. 529 Peripheral Androgen Receptors Sustain the Acrobatics and Fine Motor Skill of $530 \quad$ Elaborate Male Courtship. Endocrinology 154:3168-3177.

531 Fuxjager, M. J. and B. A. Schlinger. 2015. Perspectives on the evolution of animal 532 533 dancing: a case study of manakins. Current Opinion in Behavioral Sciences 6:712.

534 Fuxjager, M.J., J.D. Schultz, J. Barske, N.Y. Feng, L. Fusani, A. Mirzatoni, et al. 2012. 535 Spinal Motor and Sensory Neurons Are Androgen Targets in an Acrobatic Bird. $536 \quad$ Endocrinology 153:3780-3791.

537 Fuxjager, M.J., J.-H. Lee, T.-M. Chan, J.H. Bahn, J.G. Chew, X. Xiao, et al. 2016.

538 Research Resource: Hormones, genes and athleticism: effect of androgens on the $539 \quad$ avian muscular transcriptome. Molecular Endocrinology 1270. 
540 Griffiths, R., M. C. Double, K. Orr, and R. J. G. Dawson. 1998. A DNA test to sex most $541 \quad$ birds. Molecular Ecology 7:1071-1075.

542 Huxley JS. 1914 The Courtship- habits of the Great Crested Grebe (Podiceps cristatus);

$543 \quad$ with an addition to the Theory of Sexual Selection. Proc. Zool. Soc. Lond. 84:

544 491-562. doi:10.1111/j.1469-7998.1914.tb07052

545 Kendrick, A. M. and B. A. Schlinger. 1996. Independent Differentiation of Sexual and 546 Social Traits. Hormones and Behavior 30:600-610.

547 Lahaye, S. E. P., M. Eens, V. M. Darras, and R. Pinxten. 2012. Testosterone stimulates the 548 expression of male-typical socio-sexual and song behaviors in female budgerigars 549 (Melopsittacus undulatus): An experimental study. General and Comparative $550 \quad$ Endocrinology 178:82-88.

551 Langmore, N. E. 1998. Functions of duet and solo songs of female birds. Trends in $552 \quad$ Ecology \& Evolution 13:136-140.

553 Lank, D. B., M. Coupe, and K. E. Wynne-Edwards. 1999. Testosterone-induced male 554 traits in female ruffs (Philomachus pugnax): autosomal inheritance and gender 555 differentiation. Proceedings of the Royal Society B: Biological Sciences $556 \quad 266: 2323-2330$.

557 Madison, F. N., M. L. Rouse, J. Balthazart, and G. F. Ball. 2015. Reversing song behavior 558 phenotype: Testosterone driven induction of singing and measures of song quality 

in adult male and female canaries (Serinus canaria). General and Comparative Endocrinology 215:61-75.

561 McCarthy, M.M. 2016. Multifaceted origins of sex differences in the brain. 2016. Phil. $562 \quad$ Trans. R. Soc. B 371:20150106.

563 Odom, K. J., M. L. Hall, K. Riebel, K. E. Omland, and N. E. Langmore. 2014. Female 564 song is widespread and ancestral in songbirds. Nature Communications 5.

565 Phoenix, C. H., Goy, R. W., Gerall, A. A. and Young, W. C. 1959. Organizing action of 566 prenatally administered testosterone propionate on the tissues mediating mating 567 behavior in the female guinea pig. Endocrinology 65: 369-382.

568 Price, J.J., N.R. Friedman, and K.E. Omland. 2007. Song and plumage evolution in the 569 new world orioles (icterus) show similar lability and convergence in patterns. $570 \quad$ Evolution 61:850-863.

571 Prum, R.O. 1990. Phylogenetic analysis of the evolution of display behavior in the 572 neotropical manakins (Aves, Pipridae). Ethology 84: 202-231.

573 Saldanha, C. J., M. J. Tuerk, Y.-H. Kim, A. O. Fernandes, A. P. Arnold, and B. A. 574 Schlinger. 2000. Distribution and regulation of telencephalic aromatase 575 expression in the zebra finch revealed with a specific antibody. The Journal of $576 \quad$ Comparative Neurology 423:619-630. 
577 Schlinger. B. A. 1998. Sexual differentiation of avian brain and behavior: Current Views 578 on Gonadal Hormone-Dependent and Independent Mechanisms. Annual Review 579 of Physiology 60:407-429.

580 Schlinger, B. A., J. Barske, L. Day, L. Fusani, and M. J. Fuxjager. 2013. Hormones and 581 the neuromuscular control of courtship in the golden-collared manakin (Manacus 582 vitellinus). Frontiers in Neuroendocrinology 34:143-156.

583 Tinbergen, N. 1951 The Study of Instinct. Oxford University Press, New York.

584 West-Eberhard, M.J. 2003. Developmental Plasticity and Evolution. Oxford University $585 \quad$ Press, Oxford, U.K.

586 Wiens, J. J. 1999. Phylogenetic evidence for multiple losses of a sexually selected 587 character in phrynosomatid lizards. 1999. Proceedings of the Royal Society B:

588 Biological Sciences 266:1529-1535. 
Acknowledgments

Acknowledgements

We thank the Panama Rainforest Discovery Center for graciously allowing us access to the aviary and the Panamanian MiAmbiente for granting research permits. We thank the Smithsonian Tropical Research Institute staff, especially R. Urriola and V. Fernandez, for extensive logistical support. M.

Braun and M. Fuxjager assisted with aviary use and set-up. We are grateful to M. Rensel and J.

Touchon for much help with the statistical analyses. This work was supported by NSF-IOS-0646459 to B.A.S. 
Figure 1. A) The percentage of days (mean \pm S.E.) for which courtship behavior was observed (presence or absence) by sex and treatment. T-treatment included 7 females (total of 130 observation days) and 4 juvenile males (80 observation days). Control-treatment included 4 females (84 observation days) and 3 juvenile males (63 observation days). B) Box plots of daily rate of courtship behavior by sex and treatment. Only days when a behavior was performed are included (non-zero observations), and sample sizes are shown at the bottom (number of females/total days when behavior was observed). Data points representing individual rate are superimposed on the plot.

A
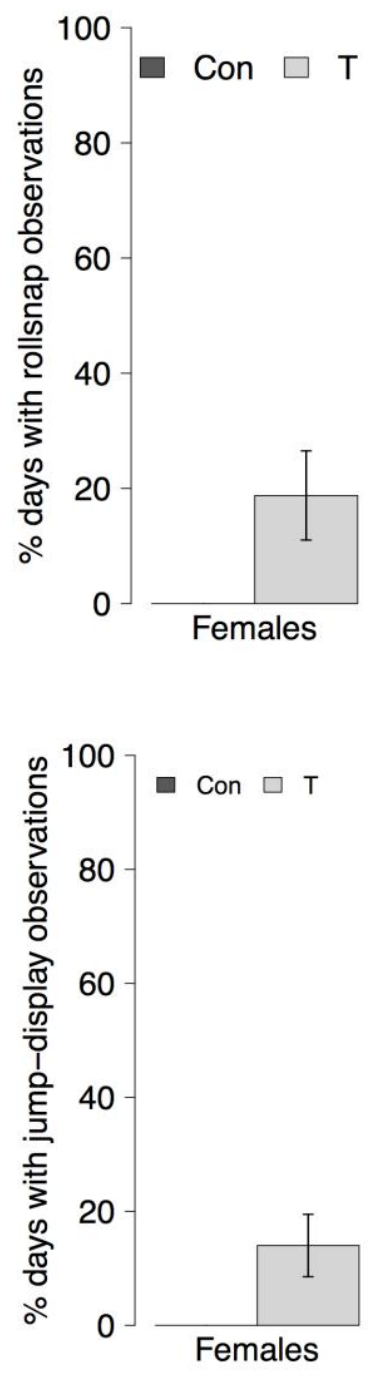

B
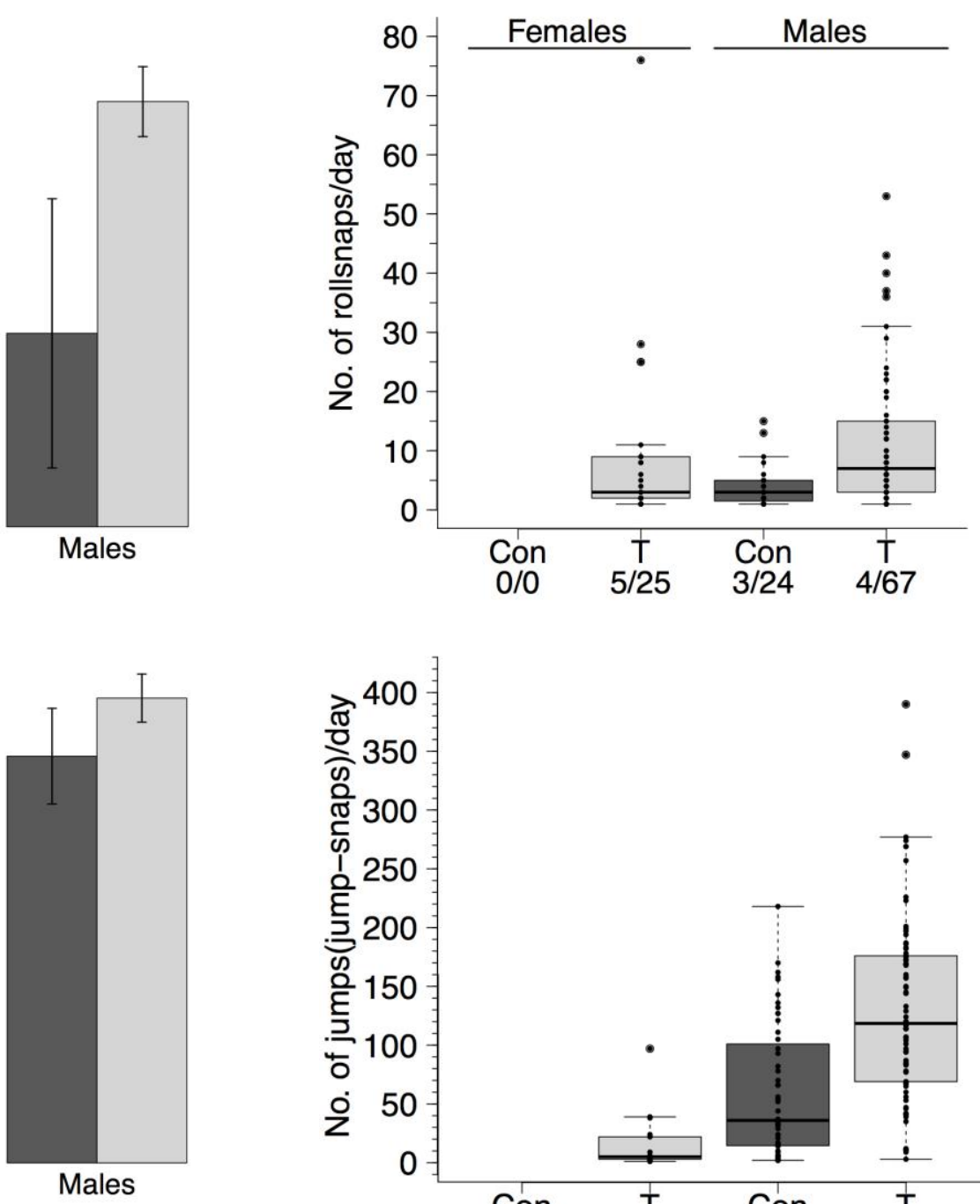

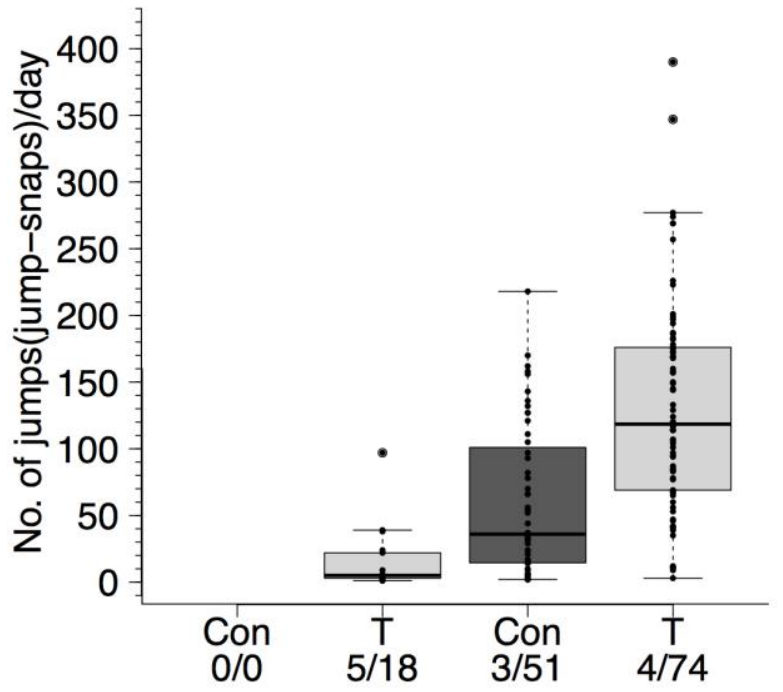


Figure 2
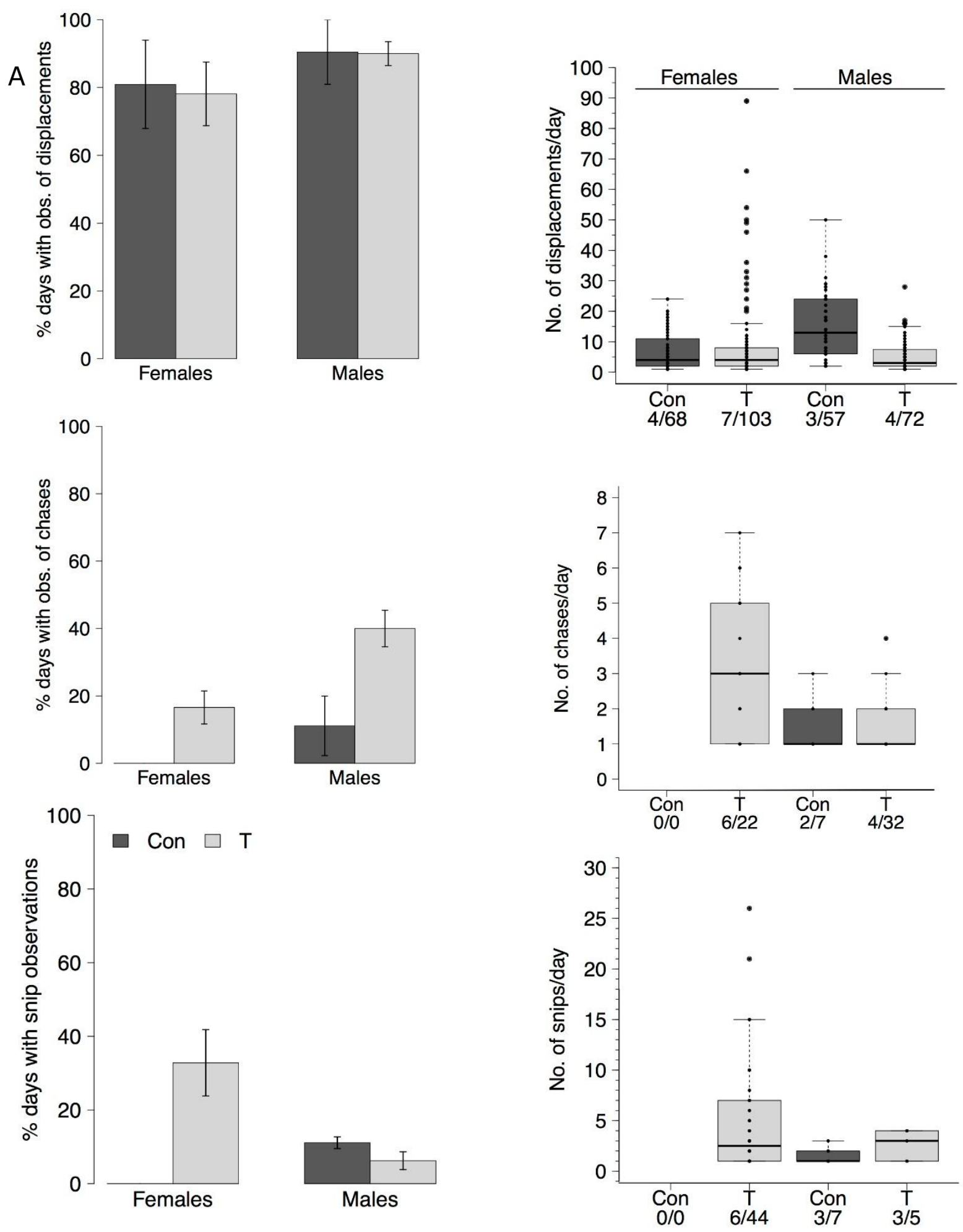

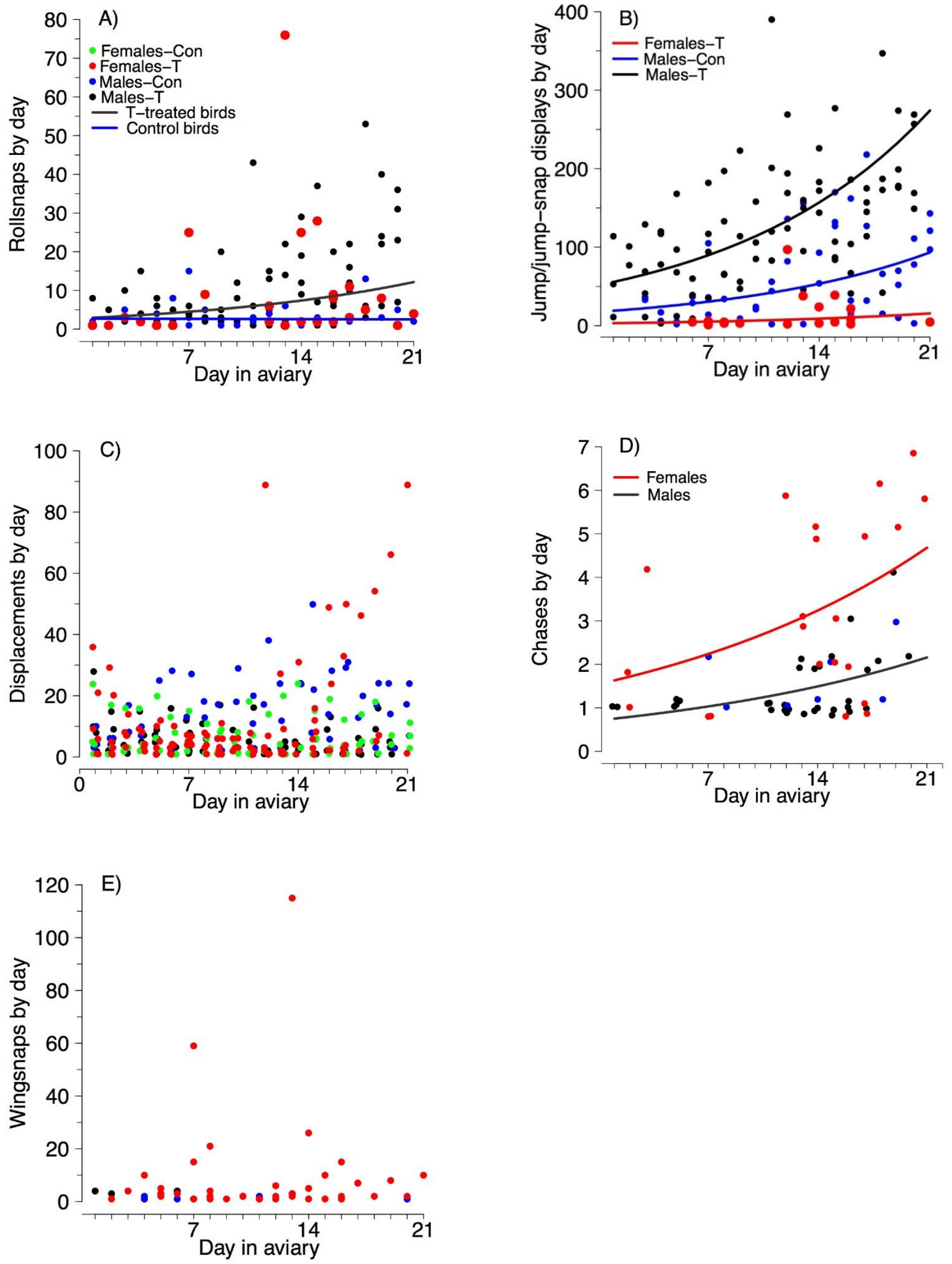